\title{
Spectral Analysis of MST Radar Signal using Maximum Likelihood Estimation Algorithm
}

\author{
G. Madhavi Latha, G. Chandraiah, S.Varadarajan, T.Sreenivasulu Reddy, P. Satish Kumar
}

\begin{abstract}
In this work, we propose Maximum likelihood estimation of low-rank Toeplitz covariance matrix (MELT) with reduced complexity algorithm for computing the power spectral density of mesosphere-stratosphere-troposphere (MST) radar data. MELT is designed based on the method of majorization-minimization and it is an iterative algorithm to update the powers in each successive step. We tested MELT algorithm for complex signal, which contain multiple frequency components in existence of different noise conditions. For simulated complex data, it can be seen that MELT works much better for low Signal to Noise Ratio (SNR) conditions and also effectively detects the frequency components with a fine resolution in the existence with high noise impact. At last, MELT algorithm is applied to the radar data received from MST radar established at National Atmospheric Research laboratory (NARL), Gadhanki. MELT algorithm estimates the accurate Doppler spectra and thus in turn, estimate the wind parameters using Doppler profiles. For the purpose of validation, the obtained radar results through MELT are compared with the Global Positioning System (GPS) radiosonde.
\end{abstract}

Keywords: Majorization-Minimization (MM) technique, Maximum-likelihood estimation (MLE), Toeplitz matrix, Spectrum estimation, MST Radar and GPS radiosonde.

\section{INTRODUCTION}

To forecast the dynamic changes in atmosphere, we require regular monitoring of wind velocities at different altitudes. The GPS radiosonde is the standard method of monitoring the atmospheric parameters like pressure, humidity, temperature, wind speed and the wind direction. Over the last three decades, ground based pulsed Doppler radars working in the frequency bands of VHF and UHF has become popular. The Doppler radar receiver performs Doppler processing on the echo signal backscattered due to refractive index fluctuations. The relative backscattered intensity and wind velocity is determined based on Received signal power, Doppler shift and Spectral width. Signal processing system in the receiver separates the echo samples on the basis of 'time of arrival'. The echo signals arriving at different time instants are processed separately as they are from different sections of the range. These section divisions of the radar range are known as 'Range-bins'. The MST radar is placed at NARL, Gadhanki $\left(13.47^{\circ} \mathrm{N}, 13.79 .18^{\circ} \mathrm{E}\right)$.

Revised Manuscript Received on December 30, 2019.

* Correspondence Author

G. Madhavi Latha, ECE, S.V. Engineering College, Tirupati, India Email: gmlatha2009@gmail.com

G. Chandraiah*, ECE, S.V. Engineering College, Tirupati, India. Email: gchandraece38@gmail.com

S.Varadarajan, ECE, S.V. Engineering College, Tirupati, India.. Email: varadasouri@gmail.com

(C) The Authors. Published by Blue Eyes Intelligence Engineering and Sciences Publication (BEIESP). This is an open access article under the CC BY-NC-ND license (http://creativecommons.org/licenses/by-nc-nd/4.0/)
MST radar provides wind data in the form of range bins, every range-bin with a height of $150 \mathrm{~m}$ and starting from a height of $3.6 \mathrm{~km}$. Doppler beam swinging (DBS) technique can be used in MST radar for collecting wind information in multiple beam directions such as West, East, Zenith-X, Zenith-Y, North, and south and every beam contains 150 range-bins. In this application, each range-bin has 512 complex data samples. To estimate Doppler profile, we computed power spectrum for each bin in a particular beam. Once Doppler profile is estimated, Doppler velocities are obtained by multiplying Doppler frequency with $c / 2 f_{\mathrm{C}}$. Where, $f_{\mathrm{C}}$ indicates the radar operating frequency and $c$ is light velocity. In literature, authors presented different algorithms for estimating Doppler spectrum for MST data. An adaptive estimation method is exhibited in [1] to evaluate the Doppler spectrum. This method has some parameters were utilized to adaptively fallow the radar signal within the range-Doppler spectral frame. Bi-spectral estimation [2] and Multi taper spectral estimation [3] is used to radar signal, which include broadened spectral peak and high computational cost respectively. Other algorithms like cepstral thresholding [4], wavelet-based denoising [5] and PCA approach [6] are also applied to spectrum estimation of the MST data. MELT algorithm is based on robust covariance estimation is explained in [7], and it is found to give improved results in both simulation and radar signals.

The remaining paper is ordered as follows. In Section 2, the simulation 'data model is formulated and introduces the proposed (MELT) algorithm. Section 3, provides simulation and radar results to demonstrate the usefulness of MELT algorithm. In the end, we conclude the paper in section 4.

Notations: Throughout the article, matrices are representing by bold uppercase letters and vectors are indicating by bold lowercase letters. Italic letters represent scalars. Superscript $(\cdot)^{T},(\cdot)^{H}$ denote transpose and Complex conjugate transpose respectively. $\operatorname{tr}(\cdot)$ indicating trace of the square matrix. $|\cdot|$ and $\|\cdot\|$ denote the modulus and Frobenius norm respectively. $E(\cdot)$ indicates the expectation operation. Finally diag $(\cdot)$ indicates diagonal matrix.

\section{MELT}

The signal model and steps concerned in implementation of MELT algorithm is presented in this section. Let us consider a data model; it consists of C Complex exponential terms in noise

$$
x(n)=\sum_{m=1}^{C} q_{m} e^{j \Omega_{m} n}+\varepsilon(n)
$$


where $q_{m}=\left|q_{m}\right| e^{j \varphi_{m}}$

and the phases $\varphi_{m}$ are distributed uniformly over $[0,2 \pi]$. Considering the signal $x(n)$ at its current and future M-1 values, it can be represented in vector form as

$$
\boldsymbol{x}(n)=\left[\begin{array}{llll}
x(n) & x(n+1) & \cdots & x(n+M-1)
\end{array}\right]^{T}
$$

(3)

$\boldsymbol{x}(n)=\sum_{m=1}^{C} q_{m} \boldsymbol{v}\left(\Omega_{m}\right) e^{j \Omega_{m} n}+\boldsymbol{\varepsilon}(n)$

Where

$$
\varepsilon(n)=\left[\begin{array}{llll}
\varepsilon(n) & \varepsilon(n+1) & \cdots & \varepsilon(n+M-1)
\end{array}\right]^{T}
$$

and

$$
\boldsymbol{v}(\Omega)=\left[\begin{array}{lllll}
1 & e^{j \Omega} & e^{j 2 \Omega} & \cdots & e^{j(M-1) \Omega}
\end{array}\right]
$$

$\boldsymbol{v}(\Omega)$ denote a M-point DFT vector at frequency $\Omega$. For the data model considered in (3) the auto-correlation matrix (ACM) of the data model can be written as

$$
\begin{aligned}
& \boldsymbol{R}=E\left[\begin{array}{ll}
\boldsymbol{x}(n) & \boldsymbol{x}^{H}(n)
\end{array}\right] \\
& =\sum_{m=1}^{C}\left|q_{m}\right|^{2} \boldsymbol{v}\left(\Omega_{m}\right) \boldsymbol{v}^{H}\left(\Omega_{m}\right)+\sigma_{\varepsilon}^{2} \boldsymbol{I}
\end{aligned}
$$

(7)

$$
=\boldsymbol{V} \boldsymbol{\Lambda} \boldsymbol{V}^{H}+\sigma_{\varepsilon}^{2} \boldsymbol{I}=\boldsymbol{R}_{s}+\boldsymbol{R}_{\varepsilon}
$$

$$
\text { where } \boldsymbol{V}=\left[\begin{array}{llll}
v\left(\Omega_{1}\right) & v\left(\Omega_{2}\right) & \cdots & v\left(\Omega_{C}\right)
\end{array}\right]
$$

(9)

is a $M \times C$ matrix and

$$
\Lambda=\left[\begin{array}{cccc}
\left|q_{1}\right|^{2} & 0 & \cdots & 0 \\
0 & \left|q_{2}\right|^{2} & \cdots & 0 \\
\vdots & \vdots & \ddots & \vdots \\
0 & 0 & \cdots & \left|q_{C}\right|^{2}
\end{array}\right]
$$

is a $C \times C$ diagonal matrix with diagonal elements being the powers associated with exponentials. The $\boldsymbol{R}_{\varepsilon}=\sigma_{\varepsilon}^{2} \boldsymbol{I}$ is a full rank matrix, where as $\boldsymbol{R}_{s}=\boldsymbol{V} \boldsymbol{\Lambda} \boldsymbol{V}^{H}$ is a rank-deficient matrix for $C<M$.

From the observations $\boldsymbol{x}(n)$ and the model as in (8), the problem is to estimate the frequencies $(\Omega \mathrm{m})$, their corresponding powers $\left|q_{m}\right|^{2}$ and the noise variance $\sigma_{\varepsilon}^{2}$. Let us call the parameter set as $\left(\boldsymbol{\theta}, \sigma_{\varepsilon}^{2}\right)$ Note that in development of the model we are taking into consideration the hypothetical or accurate correlation matrix $\boldsymbol{R}$. However, in practice it has to be estimated from the measured data samples. To increase the estimation accuracy of the frequencies, let us modify (8) as

$$
\boldsymbol{R}=\boldsymbol{F P} \boldsymbol{F}^{H}+\sigma_{\varepsilon}^{2} \boldsymbol{I}
$$

where $\boldsymbol{F}$ is $(\mathrm{M} \times \mathrm{L})$ DFT matrix with $\boldsymbol{F}=\left[\begin{array}{ll}\boldsymbol{I} & \boldsymbol{O}\end{array}\right] \tilde{\boldsymbol{F}}$, where $\boldsymbol{I}$ is identity matrix of size $(\mathbf{M} \times \mathbf{M})$ and $\boldsymbol{O}$ is all zero matrix of size $(\mathrm{M} \times \mathrm{L}-\mathrm{M})$.

$\tilde{F}_{m, n}=\frac{1}{\sqrt{L}} e^{\frac{-j 2 \pi m, n}{L}}, m \& n\{=0$ to $L-1$

$\boldsymbol{P}$ is $(\mathrm{L} \times \mathrm{L})$ diagonal matrix with

$[\boldsymbol{P}]_{m, n}=p_{m}=\left|q_{m}\right|^{2}>0 \& \boldsymbol{p}=\left[\begin{array}{llll}p_{1}, & p_{2}, & \cdots, & p_{L}\end{array}\right]^{T}$

with $\|\boldsymbol{p}\|_{0}=C$. Using the time window vector from (2) we can from the data matrix $\mathrm{X}$ as

$$
\boldsymbol{X}=\left[\begin{array}{c}
\boldsymbol{x}^{T}(0) \\
\boldsymbol{x}^{T}(1) \\
\vdots \\
\boldsymbol{x}^{T}(N-1)
\end{array}\right]=\left[\begin{array}{cccc}
x(0) & x(1) & \cdots & x(M-1) \\
x(1) & x(2) & \cdots & x(M) \\
\vdots & \vdots & \cdots & \vdots \\
x(n) & x(n+1) & \cdots & x(n+M-1) \\
\vdots & \vdots & \cdots & \vdots \\
x(N-1) & x(N) & \cdots & x(N+M-2)
\end{array}\right]
$$

$\mathrm{X}$ has dimensions with $N \times M$, where $N$ and $M$ representing the data vector and time-window lengths respectively. From $\mathbf{X}$ the sample correlation matrix can be estimated as $\boldsymbol{R}=\frac{1}{N} \boldsymbol{X}^{H} \boldsymbol{X}$

\section{A. Maximum Likelihood Estimation (ML)}

The ML method is a benchmark technique in the field of statistical estimation hypothesis. The ML estimates are those values of the unknown parameters that maximize the likelihood function of the observed data. This can be interpreted as finding the set of parameters that make the observed data most probable. The observed data is modelled as stationary statistic process, possessing Gaussian distribution, as it is enthused by the central limit theorem. Under the assumption that the observation process $\boldsymbol{x}(n)$, constitutes the zero-mean Gaussian random and stationary, the auto correlation matrix of data can be written as

$$
\boldsymbol{R}=E\left[\boldsymbol{x}(n) \boldsymbol{x}^{H}(n)\right]
$$

The likelihood function of single observation $x(n)$, is

$$
f(\boldsymbol{x}(\mathrm{n}))=\frac{1}{\pi^{M}|\boldsymbol{R}|} e^{-\boldsymbol{X}(\mathrm{n})^{H} \boldsymbol{R}^{-1} \boldsymbol{x}(\mathrm{n})}
$$

where $|\boldsymbol{R}|$ is the determinant of $\boldsymbol{R}$. It is the complex Multi-variate Gaussian distribution. Under the assumption, the snapshots are independent as well as identically distributed; the likelihood of the data set $\boldsymbol{x}(0), \boldsymbol{x}(1) \ldots \boldsymbol{x}(N-1)$ is given by

$$
\begin{aligned}
& f\left(\boldsymbol{x}(0), \ldots, \boldsymbol{x}(N-1) \mid \boldsymbol{\theta}, \sigma_{\mathcal{E}}^{2}\right)= \\
& \prod_{n=0}^{N-1} \frac{1}{\pi^{M}|\boldsymbol{R}|} e^{-\boldsymbol{x}^{H}(n) \boldsymbol{R}^{-1} \boldsymbol{x}(n)}
\end{aligned}
$$


Maximizing $f\left(\boldsymbol{\theta}, \sigma_{\varepsilon}^{2}\right)$ is corresponding to minimizing the negative log-likelihood function.

$$
\begin{aligned}
& -\log \left(f\left(\boldsymbol{\theta}, \sigma_{\varepsilon}^{2}\right)\right)=-\sum_{n=0}^{N-1} \log \left(\frac{1}{\pi^{M}|\boldsymbol{R}|} e^{-\boldsymbol{X}^{H}(n) \boldsymbol{R}^{-1} \boldsymbol{X}(n)}\right) \\
& =M N \log \pi+N \log |\boldsymbol{R}|+\sum_{n=0}^{N-1} \boldsymbol{x}^{H}(n) \boldsymbol{R}^{-1} \boldsymbol{x}(n)
\end{aligned}
$$

Ignoring the constant term in (19) and normalizing by $\mathrm{N}$, the ML estimate is equivalent to solving the subsequent optimization problem.

$$
\left[\hat{\boldsymbol{\theta}}, \hat{\sigma}_{\varepsilon}^{2}\right]=\underset{\boldsymbol{\theta}, \sigma_{\varepsilon}^{2}}{\arg \min _{\varepsilon}} l\left(\boldsymbol{\theta}, \sigma_{\varepsilon}^{2}\right)
$$

where

$$
l\left(\boldsymbol{\theta}, \sigma_{\varepsilon}^{2}\right)=\log |\boldsymbol{R}|+\frac{1}{N} \sum_{n=0}^{N-1} \boldsymbol{x}^{H}(n) \boldsymbol{R}^{-1} \boldsymbol{x}(n)
$$

$$
=\log |\boldsymbol{R}|+\operatorname{Tr}\left\{\boldsymbol{R}^{-1} \hat{\boldsymbol{R}}\right\}
$$

where

$$
\hat{\boldsymbol{R}}=\frac{1}{N} \sum_{n=0}^{N-1} \boldsymbol{x}(n) \boldsymbol{x}^{H}(n)=\frac{1}{N} \boldsymbol{X}^{H} \boldsymbol{X}
$$

The ML estimation of the parameters $\left(\boldsymbol{\theta}, \sigma_{\varepsilon}^{2}\right)$, which are the frequencies, the powers of the exponentials and noise power can be observed as ML estimation of $\mathbf{R}$. In our problem $\mathbf{R}$ is characterized by $\boldsymbol{R}=\boldsymbol{R}_{s}+\sigma_{\varepsilon}^{2} \boldsymbol{I}$. where $\boldsymbol{R}_{s}$ is a low-rank Toeplitz matrix and $\sigma_{\varepsilon}^{2}>0$. The noise power $\sigma_{\varepsilon}^{2}$ can be calculate usig standard procedures [8]. It is difficult to solve the ML estimation problem because the objective function is non-convex in nature. In this paper an iterative approach using majorization-minimization (MM) technique is used to solve the ML estimation problem of

$$
\min _{R} \log |\boldsymbol{R}|+\operatorname{Tr}\left[\boldsymbol{R}^{-1} \hat{\boldsymbol{R}}\right]
$$

Subject to $\boldsymbol{R}=\boldsymbol{R}_{\boldsymbol{s}}+\sigma_{\varepsilon}^{2} \boldsymbol{I}$

$\operatorname{rank}\left(\boldsymbol{R}_{\mathrm{s}}\right) \leq C$, where $C<M$ and $\boldsymbol{R}_{\mathrm{s}}$ be a Hermitian Toplitz matrix. In next section, we present the MM-iterative algorithm after a brief introduction to MM approach.

\section{B. $\mathbf{M M}$}

By considering the subsequent optimization problem $\underset{\boldsymbol{x}}{\operatorname{minimize}} f(\boldsymbol{x})$

Subject to $\boldsymbol{X} \in \mathscr{X}$ wher $\mathscr{X}$ denotes the closed convex set. $f(\boldsymbol{x})$ is continuous but too difficult to manipulate. The idea is to replace by $f(\boldsymbol{x})$ by an approximating function $u\left(\boldsymbol{x}, \boldsymbol{x}_{i}\right)$ and successively minimize it with

$$
\boldsymbol{x}_{i+1}=\underset{\boldsymbol{x} \in \mathscr{X}}{\arg \min } u\left(\boldsymbol{x}, \boldsymbol{x}_{i}\right)
$$

hoping the sequence of minimisers $\left\{\boldsymbol{x}_{i}\right\}$ will converge to optimal $\boldsymbol{x}^{*}$. The approximating function $u\left(\boldsymbol{x}, \boldsymbol{x}_{i}\right)$ is required to satisfy the following properties.

$$
\begin{aligned}
& f\left(\boldsymbol{x}_{i}\right)=u\left(\boldsymbol{x}_{i}, \boldsymbol{x}_{i}\right) \forall \boldsymbol{x}_{i} \in \mathscr{X} \\
& f(\boldsymbol{x}) \leq u\left(\boldsymbol{x}, \boldsymbol{x}_{i}\right) \forall \boldsymbol{x} \& \boldsymbol{x}_{i} \in \mathscr{X} \\
& f^{1}\left(\boldsymbol{x}_{i} ; \boldsymbol{d}\right)=\left.u^{1}\left(\boldsymbol{x}, \boldsymbol{x}_{i} ; \boldsymbol{x}\right)\right|_{\boldsymbol{x}=\boldsymbol{x}_{i}} \forall \boldsymbol{d}
\end{aligned}
$$

with $\boldsymbol{x}_{i}+\boldsymbol{d} \in$

where $f^{1}\left(\boldsymbol{x}_{i} ; \boldsymbol{d}\right)$ stands for the directional derivative of $f(\cdot)$ at $\boldsymbol{x}$ along the direction $\boldsymbol{d}$. In our problem, the function to be minimized is

$$
\begin{aligned}
& f(\boldsymbol{p})=\log |\boldsymbol{R}|+\operatorname{Tr}\left[\boldsymbol{R}^{-1} \hat{\boldsymbol{R}}\right] \\
& \text { Subject to } \quad \boldsymbol{R}=\boldsymbol{F}\left[\boldsymbol{P}+\sigma_{\varepsilon}^{2} \boldsymbol{I}\right] \tilde{\boldsymbol{F}}^{H} \\
& {[\boldsymbol{P}]_{m, m}=p_{m}>0 \&\|\boldsymbol{p}\|_{0} \leq \rho}
\end{aligned}
$$

Since $\log |\boldsymbol{R}|$ is concave in $\log |\boldsymbol{R}|$ [9], given $\boldsymbol{R}_{i}$, a tighter upper-bounded is given with its tangent hyper plane passing through $\boldsymbol{R}_{i}$, i.e.

$\log |\boldsymbol{R}| \leq \log \left|\boldsymbol{R}_{i}\right|+\operatorname{Tr}\left(\boldsymbol{R}_{i}^{-1}\left(\boldsymbol{R}-\boldsymbol{R}_{i}\right)\right)$

with equality achieved at $\boldsymbol{R}=\boldsymbol{R}_{i}$. Similarly, assuming $\boldsymbol{P} \succ 0 \& \boldsymbol{R}=\boldsymbol{A} \boldsymbol{P} \boldsymbol{A}^{H}$ given estimate of $\boldsymbol{P}$ denoted by $\boldsymbol{P}_{i}$ and the equivalent $\boldsymbol{R}_{i}=\boldsymbol{A} \boldsymbol{P}_{i} \boldsymbol{A}^{H}$, the matrix $\boldsymbol{R}^{-1}=\left(\boldsymbol{A P A ^ { H }}\right)^{-1}$ can be upper bounded as

$$
\boldsymbol{R}_{i}^{-1} \boldsymbol{A} \boldsymbol{P}_{i} \boldsymbol{P}^{-1} \boldsymbol{P}_{i} \boldsymbol{A}^{H} \boldsymbol{R}_{i}^{-1} \succ\left(\boldsymbol{A P A ^ { H }}\right)^{-1}
$$

Equality being achieved for $\boldsymbol{P}=\boldsymbol{P}_{i}$. The proof of this is given in Appendix-I. Given the upper bounds of $\log |\boldsymbol{R}|$ and $\boldsymbol{R}^{-1}$, the objective function

$$
f(\boldsymbol{p})=\log |\boldsymbol{R}|+\operatorname{Tr}\left[\hat{\boldsymbol{R}} \boldsymbol{R}^{-1}\right]
$$




$$
\begin{aligned}
& \leq \log \left|\boldsymbol{R}_{i}\right|+\operatorname{Tr}\left[\boldsymbol{R}_{i}^{-1} \boldsymbol{R}\right]+\operatorname{Tr}\left[\hat{\boldsymbol{R}} \boldsymbol{R}^{-1}\right]-M \\
& \leq \operatorname{Tr}\left[\boldsymbol{R}_{i}^{-1} \boldsymbol{F}(\boldsymbol{P}+\sigma \boldsymbol{I}) \boldsymbol{F} H\right]+ \\
& \operatorname{Tr}\left[\hat{\boldsymbol{R}} \boldsymbol{R}_{i}^{-1} \boldsymbol{F}\left(\boldsymbol{P}_{i}+\sigma \boldsymbol{I}\right)(\boldsymbol{P}+\sigma \boldsymbol{I})^{-1} \times\left(\boldsymbol{P}_{i}+\sigma \boldsymbol{I}\right) \boldsymbol{F}^{H} \boldsymbol{R}_{i}^{-1}\right] \\
& +\log \left|\boldsymbol{R}_{i}\right|-M
\end{aligned}
$$

using the property of the trace,

$$
\begin{aligned}
& f(\boldsymbol{p}) \leq \operatorname{Tr}\left[\boldsymbol{F}^{H} \boldsymbol{R}_{i}^{-1} \boldsymbol{F}\left(\boldsymbol{P}+\sigma_{\varepsilon}^{2} \boldsymbol{I}\right)\right]+ \\
& \operatorname{Tr}\left[\left(\boldsymbol{P}_{i}+\sigma_{\varepsilon}^{2} \boldsymbol{I}\right) \boldsymbol{F}^{H} \boldsymbol{R}_{i}^{-1} \hat{\boldsymbol{R}} \boldsymbol{R}_{i}^{-1} \boldsymbol{F}\left(\boldsymbol{P}_{i}+\sigma_{\varepsilon}^{2} \boldsymbol{I}\right)\left(\boldsymbol{P}+\sigma_{\varepsilon}^{2} \boldsymbol{I}\right)^{-1}\right] \\
& +\log \left|\boldsymbol{R}_{i}\right|-M
\end{aligned}
$$

Let $\boldsymbol{a}_{i}=\operatorname{diag}\left[\boldsymbol{F}^{H} \boldsymbol{R}_{i}^{-1} \boldsymbol{F}\right]$ and

$$
\boldsymbol{b}_{i}=\operatorname{diag}\left[\left(\boldsymbol{P}_{i}+\sigma_{\varepsilon}^{2} \boldsymbol{I}\right) \boldsymbol{F}^{H} \boldsymbol{R}_{i}^{-1} \hat{\boldsymbol{R}} \boldsymbol{R}_{i}^{-1} \boldsymbol{F}\left(\boldsymbol{P}_{i}+\sigma_{\varepsilon}^{2} \boldsymbol{I}\right)\right]
$$

Hence

$$
f(\boldsymbol{p}) \leq \boldsymbol{a}_{i}^{H}\left(\boldsymbol{p}+\sigma_{\varepsilon}^{2} 1\right)+\boldsymbol{b}_{i}^{H}\left(\boldsymbol{p}+\sigma_{\varepsilon}^{2} 1\right)^{-1}+\text { constant }
$$

The optimization problem can be reformulated as $\underset{\left\{p_{i}\right\}}{\operatorname{minimize}} \boldsymbol{a}_{i}^{H}\left(\boldsymbol{p}+\sigma_{\varepsilon}^{2} I\right)+\boldsymbol{b}_{i}^{H}\left(\boldsymbol{p}+\sigma_{\varepsilon}^{2} I\right)^{-1}$

Subject to $p_{i} \geq 0 \&\|\boldsymbol{p}\|_{0} \leq M$

In (34) the function is separable in the $p_{i} \mathrm{~s}$, therefore can be minimized independently. For any index $j$, the function is $a_{i, j}\left(p_{j}+\sigma_{\varepsilon}^{2}\right)+b_{i, j}\left(p_{j}+\sigma_{\varepsilon}^{2}\right)^{-1}$ and the minimum value of $p_{j}$ is

$p_{j}^{*}=\sqrt{\frac{b_{i, j}}{a_{i, j}}}-\sigma_{\varepsilon}^{2}$

If $\sqrt{\frac{b_{i, j}}{a_{i, j}}}<\sigma_{\varepsilon}^{2}$, then $p_{j}^{*}=0$ as power cannot be negative.

The minimum power for the function will be $\frac{b_{i, j}}{\sigma_{\varepsilon}^{2}}+a_{i, j} \sigma_{\varepsilon}^{2}$.

If $\sqrt{\frac{b_{i, j}}{a_{i, j}}}>\sigma_{\varepsilon}^{2}$, the minimum power for the function will be $2 \sqrt{b_{i, j} a_{i, j}}$.

Hence the reduction in objective value when $p_{i}>0$ will be

$$
e_{i}=\frac{b_{i, j}}{\sigma_{\varepsilon}^{2}}+a_{i, j} \sigma_{\varepsilon}^{2}-2 \sqrt{a_{i, j} b_{i, j}}
$$

The entries with the C largest $e_{i}$ s will be allowed to be non zero. The implementation steps of the above algorithm are summarized in MELT algorithm.

\section{Steps for MELT Algorithm}

Require: data points $\{x(\mathrm{n})\}, \sigma$ and $C$.

Pre calculate: $\hat{\boldsymbol{R}}=\frac{1}{N} \sum_{n=0}^{N-1} \boldsymbol{x}(n) \boldsymbol{x}^{H}(n)=\frac{1}{N} \boldsymbol{X}^{H} \boldsymbol{X}$

Set $\mathrm{i}=0$ and initialize $\mathbf{p}_{\mathbf{0}}$

(1) Form the covariance matrix $\boldsymbol{R}_{\boldsymbol{i}}$ as in (11)

(2) Compute $\boldsymbol{a}_{i}=\operatorname{diag}\left[\boldsymbol{F}^{H} \boldsymbol{R}_{i}^{-1} \boldsymbol{F}\right]$ and

$$
\boldsymbol{b}_{i}=\operatorname{diag}\left[\left(\boldsymbol{P}_{i}+\sigma_{\varepsilon}^{2} \boldsymbol{I}\right) \boldsymbol{F}^{H} \boldsymbol{R}_{i}^{-1} \hat{\boldsymbol{R}} \boldsymbol{R}_{i}^{-1} \boldsymbol{F}\left(\boldsymbol{P}_{i}+\sigma_{\varepsilon}^{2} \boldsymbol{I}\right)\right]
$$

(3) Compute $\boldsymbol{p}_{i}$ according to (35) and (36) $i \leftarrow i+1$

until the convergence criterion: $\left\|\boldsymbol{p}_{i}-\boldsymbol{p}_{i-1}\right\|_{2}<10^{-3}$ is met.

\section{RESULTS AND DISCUSSIONS}

\section{A. Simulation Results}

The simulation results for the complex test signal model discussed in section II are explained in this section. The numerical values for the complex signal to test the MELT algorithm, we consider the three frequencies of $0.1450 \mathrm{~Hz}$, $0.3100 \mathrm{~Hz}$ and $0.3150 \mathrm{~Hz}$ and associated amplitudes $q_{1}=10 e^{j \varphi_{1}}, q_{2}=10 e^{j \varphi_{2}}$ and $q_{3}=10 e^{j \varphi_{3}}$ by a sampling period of $1 \mathrm{~s}$. The phase values $\left\{\varphi_{r}\right\}_{r=1}^{3}$ are independently and uniformly distributed in $[0,2 \pi]$. The test signal is generated with $N=200$ and $L=512$. The term $\varepsilon$ introduced the noise part of the signal, which is the normal white noise with 0 mean and $\sigma^{2}$ variance. Fig.1 depicts the true power spectrum of the complex signal before adding the noise. It is seen that the spectrum contains three peaks at desired frequencies of the original signal. Fig.2 (a) and (b) show the mean power spectrum of the complex test signal by using periodogram after adding the noise in two different SNR conditions of $0 \mathrm{~dB}$ and $-15 \mathrm{~dB}$. Fig.3 (a) and (b) present the mean power spectrum of the complex test signal by using SPICE method for the SNR values of $0 \mathrm{~dB}$ and -15dB. Similarly, Fig.4 (a) and (b) show the averaged power spectrum of the test signal by using MELT for same SNR conditions. To test the efficiency of MELT, the mean square error (MSE) and output SNR values are computed using SPICE and MELT algorithms. The performance of MELT is shown in Fig.5 in terms of MSE and output signal to noise ratio (SNR) improvement. In Fig. 5(a), the MSEs for various input SNR values for both SPICE and MELT are shown. Fig. 5(b) depicts the output SNR obtained using MELT and SPICE as the input SNR varies from - 15 to $15 \mathrm{~dB}$. The output SNR is computed from the power spectrum of the signal utilizing the noise level estimation method proposed by Hildebrand and Sekhon [10]. 


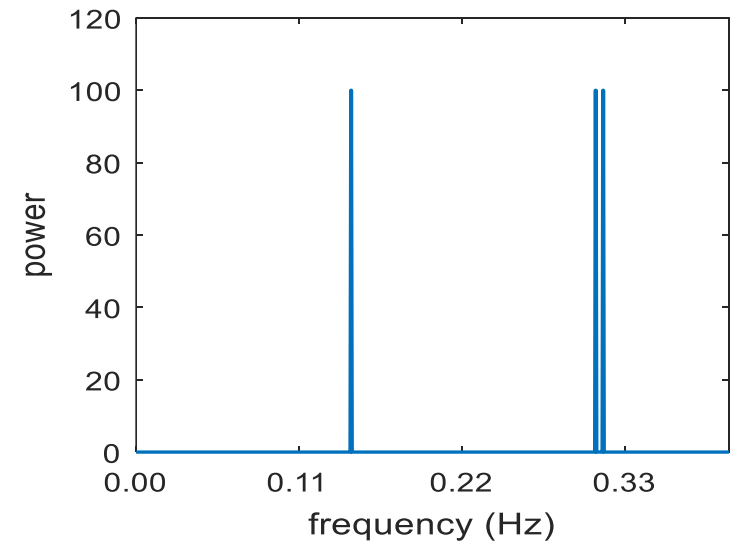

Fig.1 Power spectrum for original complex test signal
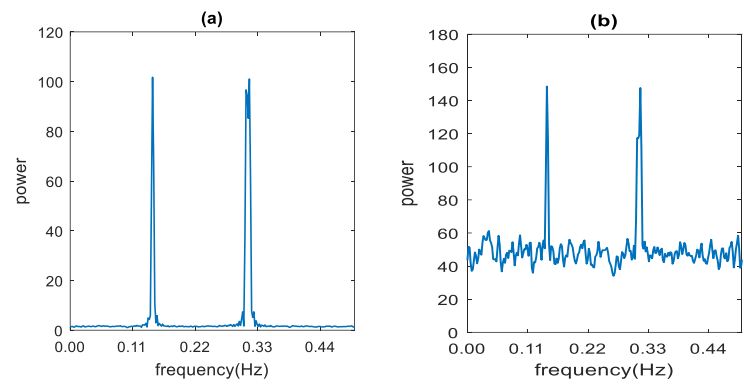

Fig.2 Power spectrum for the complex test signal using periodogram (a) $\mathrm{SNR}=0 \mathrm{~dB}$ and $(\mathrm{b}) \mathrm{SNR}=-15 \mathrm{~dB}$. (a)

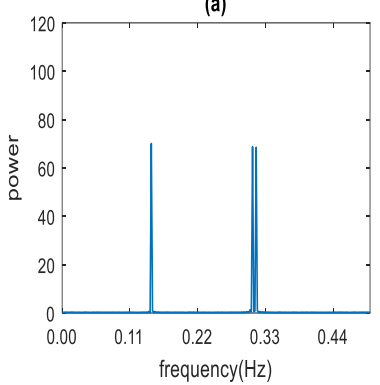

(b)

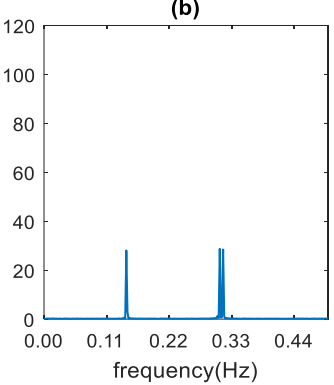

Fig.3 Power spectrum of the complex signal with SPICE (a) $\mathrm{SNR}=0 \mathrm{~dB}$ and (b) $\mathrm{SNR}=-15 \mathrm{~dB}$
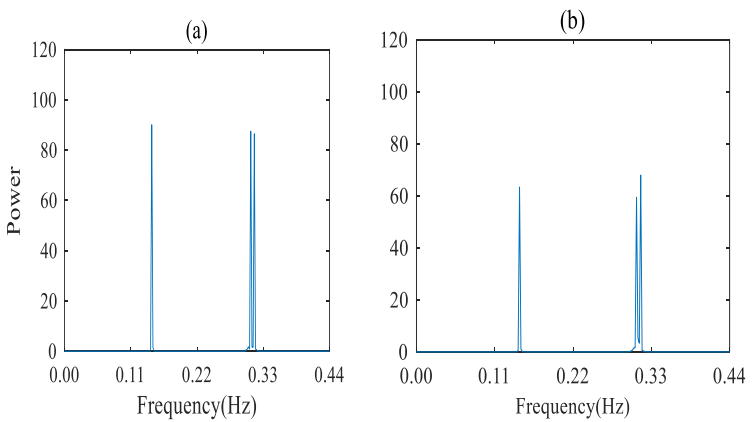

Fig.4 Power spectrum of the complex signal via MELT for (a) $\mathrm{SNR}=0 \mathrm{~dB}$ and (b) $\mathrm{SNR}=-15 \mathrm{~dB}$.
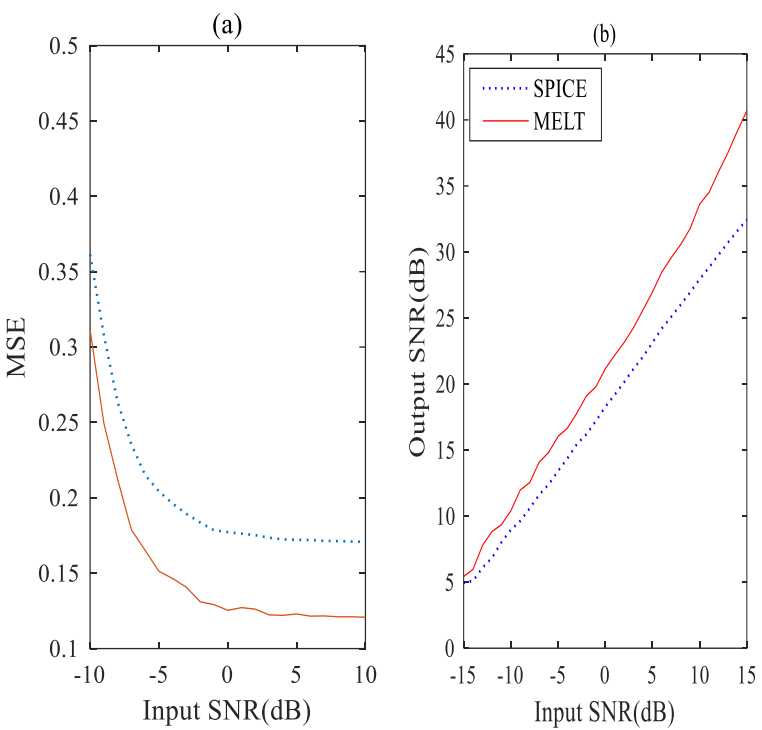

Fig.5 (a) Mean square error (MSE) and (b) Output SNR of the complex test signal using MELT.

\section{B. MST Radar Data Results}

As aforementioned in section 1, the MST radar data collected on $11^{\text {th }}$ Feb, 2015 from NARL, Gadhanki. The complex radar data with 512 points is applied to SPICE and MELT algorithms for estimating the Doppler power spectrum and applied maximum detection method to extract maximum magnitude of the single peak frequency and its index. The same process is repeated for all 150 bins and for all beams in a scan. In MELT algorithm, for the MST radar data $\boldsymbol{\sigma}$ denotes the estimated noise power [11] and $C=1$. Where $C$ is the number of nonzero frequency component in the Doppler power spectrum, i.e., single Doppler frequency is estimating at a particular bin.

\section{Methodology for computing wind components:}

Let $\mathbf{I}$ representing the frequency peaks indices for all range bins of a particular beam. Doppler shift and Doppler velocities are calculated as:

$$
\mathbf{f}=\left(\boldsymbol{I}-\frac{N F F T}{2}-6\right) \times\left(\frac{7.56+7.8}{N F F T-1}\right)
$$

$$
\mathbf{v}=\left(\boldsymbol{I}-\frac{N F F T}{2}-6\right) \times 0.029 \times \lambda
$$

where NFFT be the Fast Fourier transform (FFT) computation points and $\lambda=c / f_{c}, f_{c}$ be the radar operating frequency with $53 \mathrm{MHz}$ and $\mathrm{c}$ is the light velocity.The Doppler frequencies $\mathbf{f}_{E}, \mathbf{f}_{W}, \mathbf{f}_{Z x}, \mathbf{f}_{Z y}, \mathbf{f}_{N}, \mathbf{f}_{S}$ and Doppler velocities $\mathbf{v}_{E}, \mathbf{v}_{W}, \mathbf{v}_{Z x}, \mathbf{v}_{Z y}, \mathbf{v}_{N}, \mathbf{v}_{S}$ are calculated for all six beams. The generalized formula [1] for finding wind velocities for each bin is given as: 


\section{Spectral Analysis of MST Radar Signal using Maximum Likelihood Estimation Algorithm}

$$
\begin{gathered}
{\left[\begin{array}{c}
v_{x} \\
v_{y} \\
v_{z}
\end{array}\right]=\left[\begin{array}{ccc}
\sum_{j} \cos ^{2} \theta_{x j} & \sum_{j} \cos \theta_{x j} \cos \theta_{y j} & \sum_{j} \cos \theta_{x j} \cos \theta_{z j} \\
\sum_{j} \cos \theta_{x j} \cos \theta_{y j} & \sum_{j} \cos ^{2} \theta_{y j} & \sum_{j} \cos \theta_{y j} \cos \theta_{z j} \\
\sum_{j} \cos \theta_{x j} \cos \theta_{z j} & \sum_{j} \cos ^{2} \theta_{y j} \cos \theta_{z j} & \sum_{j} \cos ^{2} \theta_{z j}
\end{array}\right]^{-1}} \\
*\left[\begin{array}{c}
\sum_{j} v_{j} \cos \theta_{x j} \\
\sum_{j} v_{j} \cos \theta_{y j} \\
\sum_{j} v_{j} \cos \theta_{z j}
\end{array}\right]
\end{gathered}
$$

where $j$ denoting the beam number, $v_{j}$ be the radial velocity and $\theta_{x j}, \theta_{y j}$ and $\theta_{z j}$ are the radar beam making angles with the axes $X, Y$ and $Z$ with respect to different beams. The equation (39) can be written as:

$\left[\begin{array}{l}\boldsymbol{v}_{x} \\ \boldsymbol{v}_{y} \\ \boldsymbol{v}_{z}\end{array}\right]=\left[\begin{array}{ccc}0.603 & 0 & 0 \\ 0 & 0.603 & 0 \\ 0 & 0 & 0.603\end{array}\right]^{-1} *\left[\begin{array}{c}0.1736\left(\boldsymbol{v}_{E}-\boldsymbol{v}_{W}\right) \\ 0.1736\left(\boldsymbol{v}_{N}-\boldsymbol{v}_{S}\right) \\ 0.1736\left(\boldsymbol{v}_{Z X}-\boldsymbol{v}_{Z Y}\right)\end{array}\right](40)$ where $v_{x}, v_{y}$ and $v_{z}$ are denoting the zonal, meridional, and vertical wind fields respectively. Vertical velocity component $v_{z}$ is not used in the calculation of atmospheric wind speed. Hence the wind speed can be calculated as follows.

$W=\left(v_{x}^{2}+v_{y}^{2}\right)^{1 / 2}$

The estimated atmospheric wind velocities using MELT are authenticating with the wind velocities of GPS data [12].
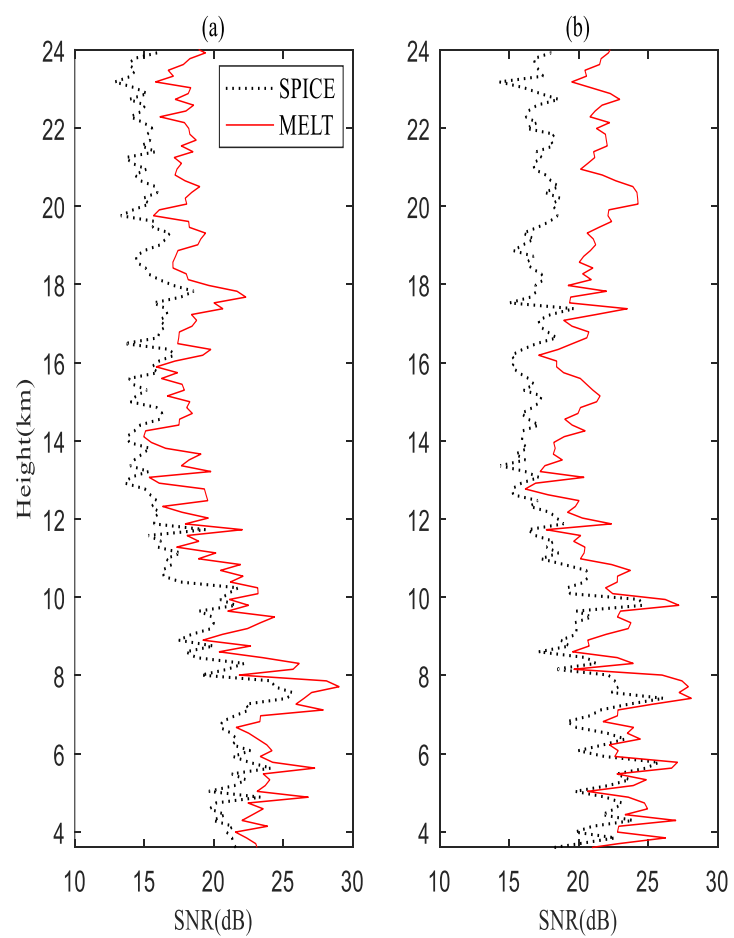

Fig.6 Height profiles of SNR estimated using MELT and SPICE of data on 5 July 2014 (a) the east beam (b) the west beam.
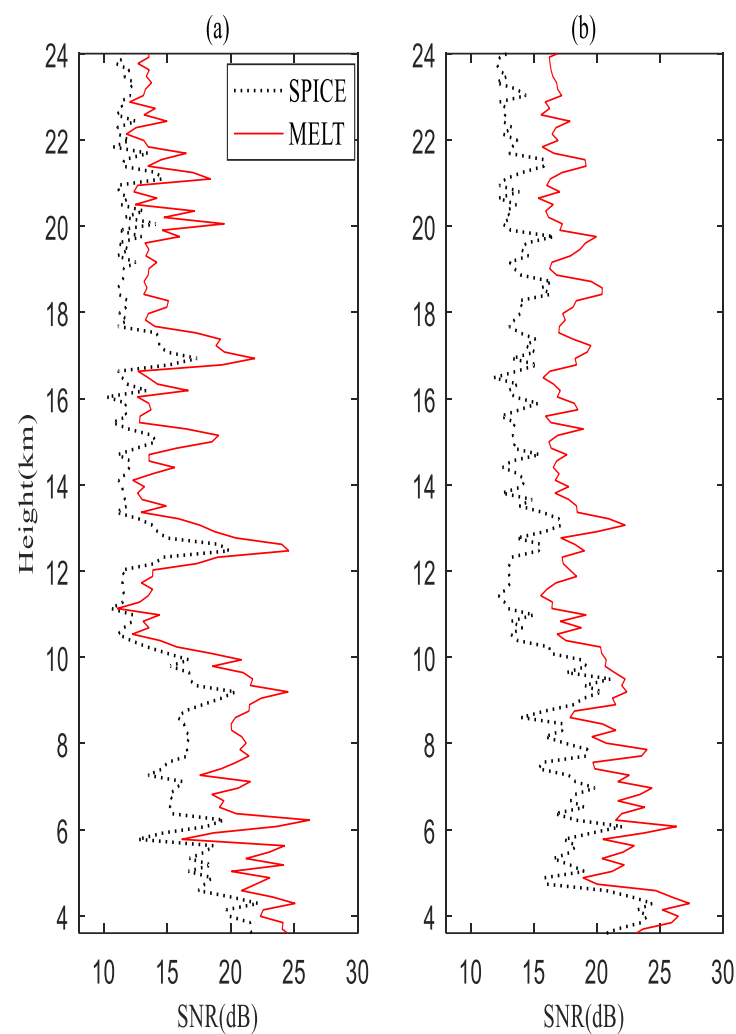

Fig.7 Height profiles of SNR estimated using MELT and SPICE for radar data collected on $11^{\text {th }} \mathrm{Feb}, 2015$ (a) East (b) West beams.

Fig. 6(a), (b) and Fig. 7(a), (b) presents the SNR profiles[13] with respect to height obtained from the spectrum estimated using SPICE and MELT for the east and west beams of data collected on $05^{\text {th }}$, July 2014 and $11^{\text {th }}$, Feb 2015 respectively. From the Figures 6 and 7, it is noted that MELT yields better SNR improvement than that of SPICE. Typical spectra and Doppler frequency height profile obtained using MELT for the east beam of an aforementioned radar data are shown in Fig. 8 and Doppler velocities of all six beams are shown in Fig.9. 

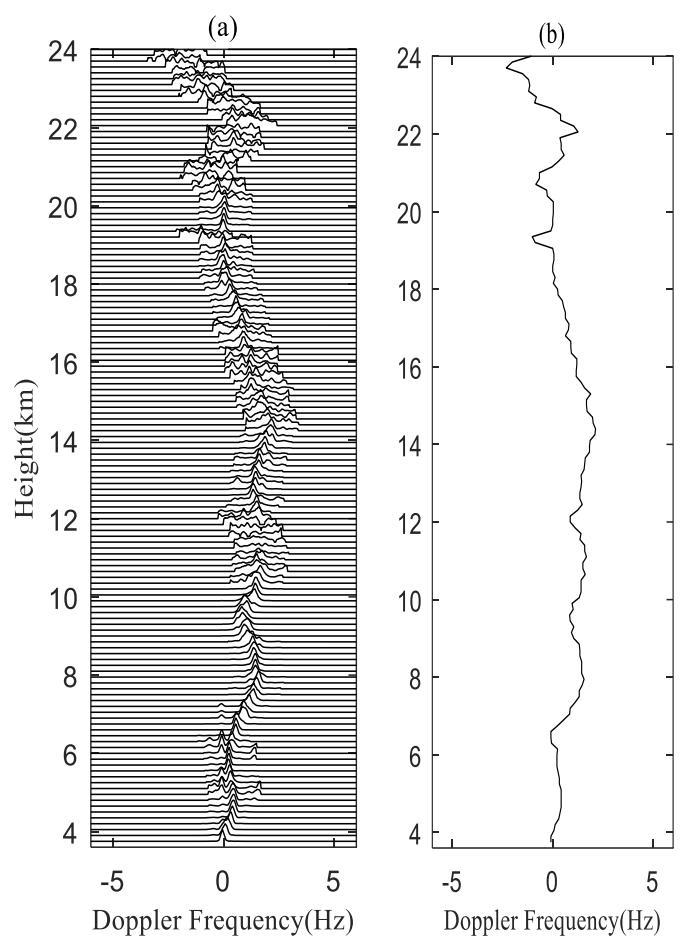

Fig.8 (a) East beam Spectra for the data received on 11 Feb 2015, (b) Doppler profile using MELT.

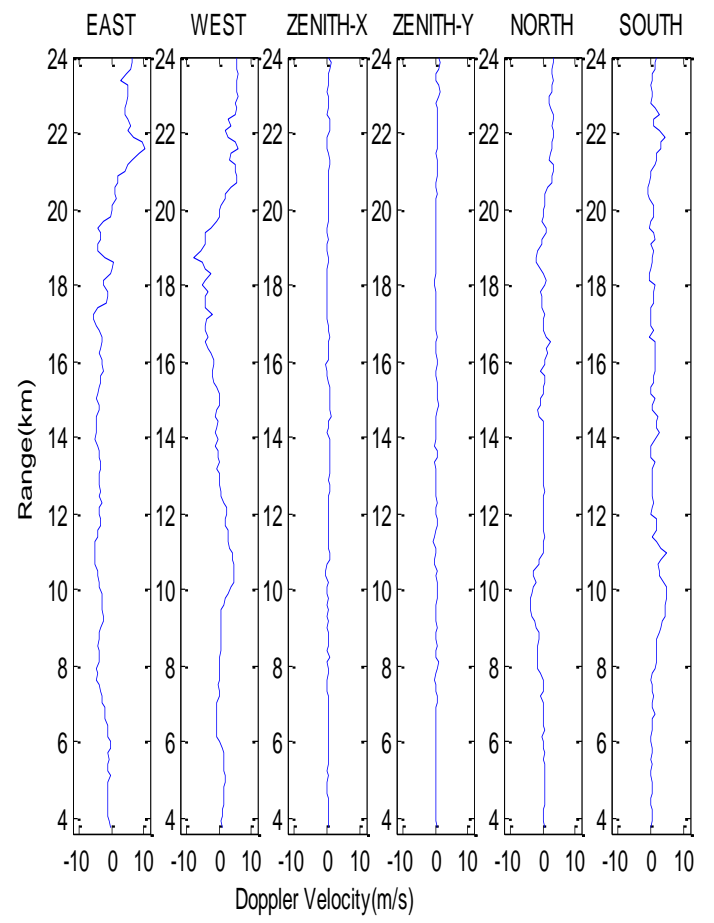

Fig.9 Doppler velocities for the radar data collected on 11 Feb 2015 using MELT.
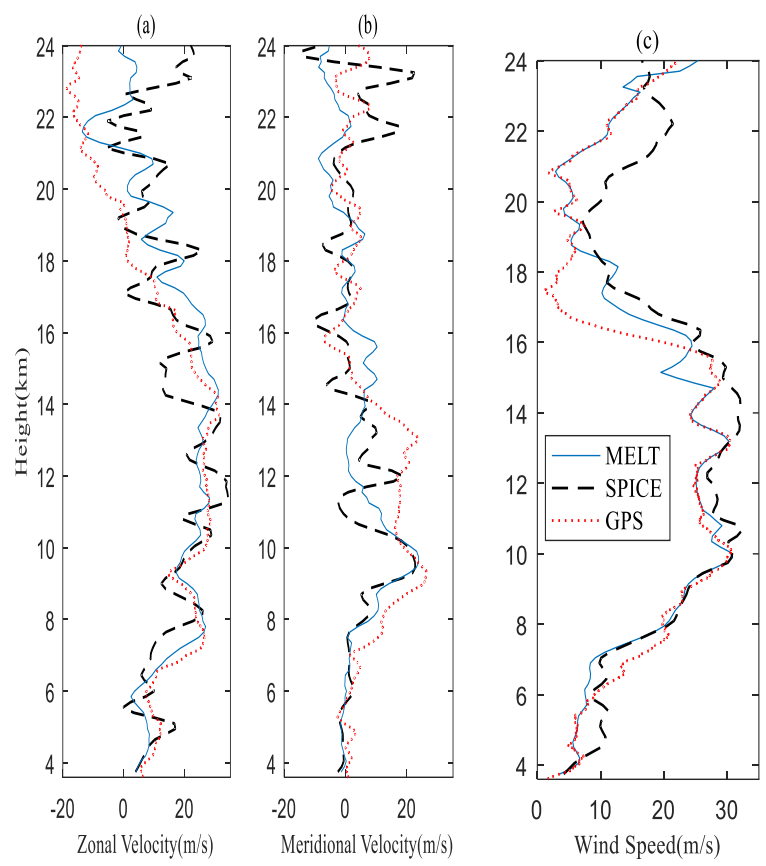

Fig.10 Comparison of Zonal, Meridional and Wind speed for the data collected on Feb 11, 2015 using MELT, SPICE and GPS radiosonde.

The zonal, meridional and wind speeds obtained from the GPS data, SPICE, and MELT are shown in Fig.10. From Fig.10, it can be seen that, the wind speed estimated using MELT is able to follow the GPS data, while the wind speed obtained using the SPICE is observed to deviate a little from those measured by GPS system.

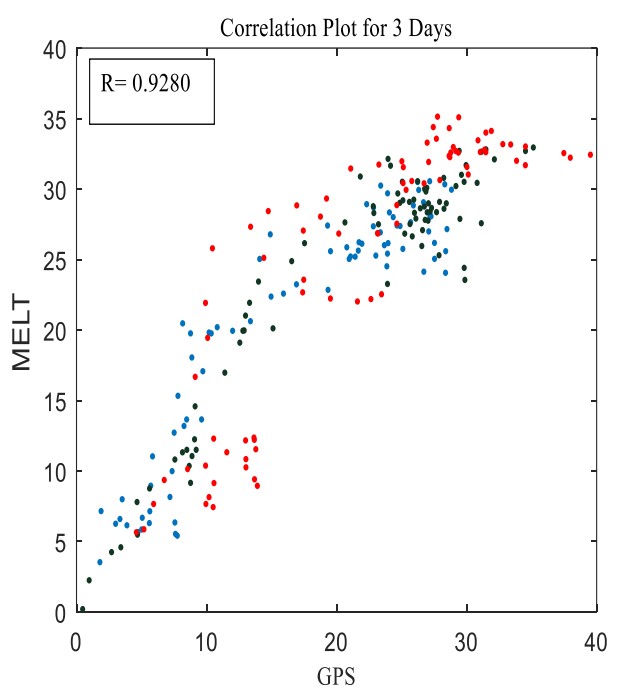

Fig.11 Correlation plot between the GPS and the MELT for the data received on Feb 11-13, 2015.

Fig.11 shows the correlation coefficient between GPS and MELT. We get the averaged correlation coefficient for three days data is 0.9280 using the MELT algorithm whereas, SPICE produces 0.8956. Therefore, MELT produces better results when compared to SPICE. 


\section{Spectral Analysis of MST Radar Signal using Maximum Likelihood Estimation Algorithm}

\section{CONCLUSION}

An algorithm based on maximum likelihood estimation to estimate the Doppler power spectrum and draw the wind profile of MST data has been presented. Moreover, MELT algorithm is based on cyclic methodology and the convergence of the objective values can be guaranteed. The performance of MELT has been analyzed numerically by computing the power spectrum, MSE and output SNR for various input SNR values. Numerical results for the simulation data have shown that the devised algorithm outperformed the existing algorithms. Finally, MELT is subjected to the radar data for the computation of Doppler spectrum. The radar results through MELT follow those of GPS wind measurements. This proves that MELT works superior to existing algorithms. The mean correlation coefficient among wind speeds obtained by GPS and MELT is presented for MST radar data received on February 11-13, 2015. For the validation purpose, the obtained radar results via MELT algorithm are compared with the GPS. The overall results indicate that MELT is seen to exhibit better performance than SPICE method. The algorithm is iterative, and the maximum computational time is taken for computing matrix inverse of $\boldsymbol{R}_{i}^{-1}(\mathrm{M} \times \mathrm{M})$ in each iteration. It is envisaged to use Gohberg-Semencul (G-S) algorithm for computing $\boldsymbol{R}_{i}^{-1}$ to diminish the computational complexity.

\section{Appendix-I}

The proof of (29) is presented below. Assume that $\boldsymbol{P} \succ 0$, from the identity

$$
\begin{aligned}
& \boldsymbol{S}=\left[\begin{array}{cc}
\boldsymbol{R}_{i}^{-1} \boldsymbol{A} \boldsymbol{P}_{i} \boldsymbol{P}^{-1} \boldsymbol{P}_{i} A^{H} \boldsymbol{R}_{i}^{-1} & \boldsymbol{I} \\
\boldsymbol{I} & \boldsymbol{A} \boldsymbol{P} \boldsymbol{A}^{H}
\end{array}\right] \\
& =\left[\begin{array}{c}
\boldsymbol{R}_{i}^{-1} \boldsymbol{A} \boldsymbol{P}_{i} \boldsymbol{P}^{-\frac{1}{2}} \\
\boldsymbol{A P}^{\frac{1}{2}}
\end{array}\right]\left[\begin{array}{ll}
\boldsymbol{P}^{-\frac{1}{2}} \boldsymbol{P}_{i} \boldsymbol{A}^{H} \boldsymbol{R}_{i}^{-1} & \boldsymbol{P}^{\frac{1}{2}} \boldsymbol{A}^{H}
\end{array}\right]
\end{aligned}
$$

It is known that $\boldsymbol{S} \succ 0$. By the schur compliment, $\boldsymbol{S} \succ 0$ is equivalent to

$$
\boldsymbol{R}_{i}^{-1} \boldsymbol{A} \boldsymbol{P}_{i} \boldsymbol{P}^{-1} \boldsymbol{P}_{i} \boldsymbol{A}^{H} \boldsymbol{R}_{i}^{-1} \succ\left(\boldsymbol{A P A ^ { H }}\right)^{-1}
$$

Hence (29) is proved.

\section{REFERENCES}

1. Anandan, V.K.; Balamuraliddhar, P.; Rao, P.B.; and Jain, A.R. (1996). A method for adaptive moment's estimation technique applied to MST radar echoes. Progress in electromagnetics research symposium. 360-365.

2. Anandan, V.K.; Pan, C.J.; Rajalakshmi, T.; and Ramachandra Reddy, G. (2004). multi taper spectral Analysis of atmospheric radar signal. Annals of Geophysics, 22(11), 3995-4003.

3. Anandan, V.K.; Ramachandra Reddy, G.; and Rao, P.B. (2001) Spectral Analysis of atmospheric signal using higher orders spectral estimation technique. IEEE Transactions in Geosciences and remote sensing. 39(9), 1890-1895.

4. Reddy, T.; and Reddy, G.R. (2010). MST radar signal processing using cepstral thresholding. IEEE Transactions in Geosciences and remote sensing 48(6), 704-2710.
5. Thatiparthi, S.; Gudheti, R.; and Sourirajan, V. (2009). MST radar signal processing using wavelet based denoising. IEEE Transactions in Geosciences and remote sensing letters. 6(4), 752-756.

6. Rao, D. U. M.; and Reddy, T. S. (2014). Atmospheric radar signal processing using principle component analysis. Digital Signal Processing. 32, 79-84.

7. Prabhu babu. (2016). MELT-Maximum-Likelihood Estimation of Low-Rank Toepltz Covariance Matrix. IEEE Signal processing letters. 23(11), 1587-1591.

8. Skolnik, I.; and Merrill, I.; Radar hand book, Mc Graw Hill publication, New York.

9. Boyd, S.; and Vandenberghe, L. (2003). Convex Optimization. Cambridge, UK: Cambridge University Press.

10. Hildebrand, P. H.; and Sekhon. (1974). Objective determination of the noise level in Doppler Spectra. Journal of Applied Meteorology. 13(7), 808-811.

11. Neetha Eappen, I.; Sreenivasulu Reddy, T.; and Ramachandra Reddy, G. (2015). Semiparametric Algorithm for Processing MST Radar Data. IEEE Transactions in Geosciences and remote sensing letters. 48(6), 1-9.

12. Jagannadha Rao, V. V. M.; Narayana Rao, D.; Venkata Ratnam, M.; Mohan, K.; and Rao, S .V. B. (2003). Mean vertical velocities measured by Indian MST radar and comparison with indirectly computed values. Journal of Applied Meteorology. 42( 4), 541-552.

13. Hooper, D.A. (1999). Signal and noise level estimation for narrow spectral width returns observed by the Indian MST radar, Radio science. 34(4), 859-870.

\section{AUTHOR'S PROFILE}

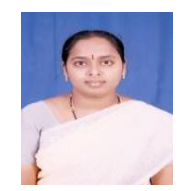

G.Madhavilatha, Research scholar, Dept. of ECE, JNTUH, Hyderabad. Research scholar in the Department of ECE at JNTUH. Hyderabad.

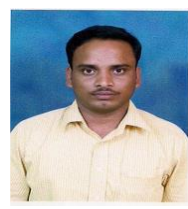

G. Chandraiah received the B.Tech degree in ECE from Jawaharlal Nehru Technological University (JNTU) in 2005 And M.Tech in Electronics Instrumentation and Communication Systems from Sri Venkateswara University College of Engineering, Tirupati in 2011. Currently, he is Ph.D full-time Scholar with the Department of Electronics and Communication Engineering, College of Engineering, Sri Venkateswara University. His research interest includes radar signal processing and signal processing.

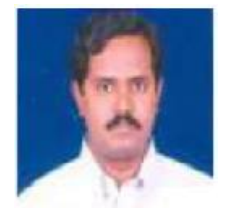

Dr. S.Varadarajan is working as Professor in Department of Electronics andCommunication Engineering, S V University College of Engineering, Tirupati,India. He received Ph.D (ECE) from S V University, Tirupati in 2003. He has 25years of teaching experience. He has widely published and presented research and technical papers in International Journals, National Journals \& conferences. Hisareas of interests include Signal \& Image Processing, Digital Communications.Systems. He is a fellow member of IETE. He chaired and served as reviewer ofnumber of international conferences and is a member of editorial board ofInternational journals. He visited USA, UK.

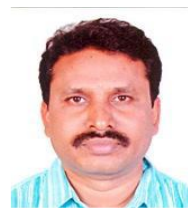

Dr.T. Sreenivasulu Reddy received the B.Tech Degree in ECE from Sri Venkateswara University, Tirupati, India, in 1990, the M.Eng. Degree in Digital Electronics from Karnataka University, Dharwad, India, in 1996 and Ph.D. degree in Radar Signal Processing from Sri Venkateswara University, Tirupati. Currently, he is Professor with the Department of Electronics and Communication Engineering, College of Engineering, Sri Venkateswara University. His research interests include radar signal processing and image processing. Mr. Reddy is a Fellow of the Institution of Electronics and Telecommunication Engineers and a member of the Indian Society for Technical Education.

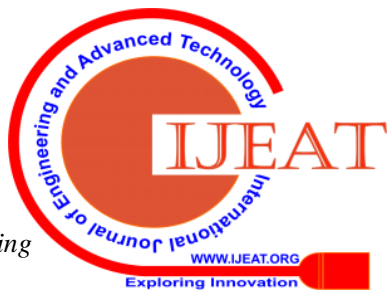


Dr. P.Satish Kumar completed B.Tech (ECE)in 1989 from NagarjunaUniversity,M.Tech(Communication Systems) in 1992 from Pondicherry Central University, Ph.D (ECE) in 2004 from JNTU, Hyderabad. He is currently working as professor in ECE department of ACE Engineering College, Ghatkesar, Hyderabad. He is guiding 10 research scholars and under his guidance one research scholar is awarded Ph.D degree. He has published 30 papers in various conferences \&journals at national \& international levels. He is Ph.D thesis evaluator for Anna University, Chennai. His research areas are Signal Processing, Computer Networking, VLSI design. He is guiding and teaching students for various competitive exams like GATE, IES \& PSU's. 